\title{
Halo histories versus Galaxy properties at $z=0-$ I. The quenching of star formation
}

\author{
Jeremy L. Tinker, ${ }^{1 \star}$ Andrew R. Wetzel, ${ }^{2,3,4}$ Charlie Conroy ${ }^{5}$ and Yao-Yuan Mao ${ }^{6}$ \\ ${ }^{1}$ Center for Cosmology and Particle Physics, Department of Physics, New York University, New York, NY 10012, USA \\ ${ }^{2}$ TAPIR, California Institute of Technology, Pasadena, CA 91101, USA \\ ${ }^{3}$ Carnegie Observatories, Pasadena, CA 91125, USA \\ ${ }^{4}$ Department of Physics, University of California, Davis, CA 95616, USA \\ ${ }^{5}$ Department of Astronomy, Harvard University, Cambridge, MA 02138, USA \\ ${ }^{6}$ Department of Physics and Astronomy and Pittsburgh Particle Physics, Astrophysics, and Cosmology Center (PITT PACC), University of Pittsburgh, \\ Pittsburgh, PA 15260, USA
}

Accepted 2017 August 8. Received 2017 August 8; in original form 2016 September 9

\begin{abstract}
We test whether halo age and galaxy age are correlated at fixed halo and galaxy mass. The formation histories, and thus ages, of dark matter haloes correlate with their large-scale density $\rho$, an effect known as assembly bias. We test whether this correlation extends to galaxies by measuring the dependence of galaxy stellar age on $\rho$. To clarify the comparison between theory and observation, and to remove the strong environmental effects on satellites, we use galaxy group catalogues to identify central galaxies and measure their quenched fraction, $f_{\mathrm{Q}}$, as a function of large-scale environment. Models that match halo age to central galaxy age predict a strong positive correlation between $f_{\mathrm{Q}}$ and $\rho$. However, we show that the amplitude of this effect depends on the definition of halo age: assembly bias is significantly reduced when removing the effects of splashback haloes - those haloes that are central but have passed through a larger halo or experienced strong tidal encounters. Defining age using halo mass at its peak value rather than current mass removes these effects. In Sloan Digital Sky Survey data, at $M_{*} \gtrsim 10^{10} \mathrm{M}_{\odot} h^{-2}$, there is a $\sim 5$ per cent increase in $f_{\mathrm{Q}}$ from low-to-high densities, which is in agreement with predictions of dark matter haloes using peak halo mass. At lower stellar mass there is little to no correlation of $f_{\mathrm{Q}}$ with $\rho$. For these galaxies, age matching is inconsistent with the data across the range of halo formation metrics that we tested. This implies that halo formation history has a small but statistically significant impact on quenching of star formation at high masses, while the quenching process in low-mass central galaxies is uncorrelated with halo formation history.
\end{abstract}

Key words: galaxies: clusters: general-galaxies: evolution - galaxies: groups: generalcosmology: observations.

\section{INTRODUCTION}

The abundance matching model for connecting galaxies to haloes has proven to be an exceptional tool for understanding both galaxy bias and galaxy evolution (see e.g. Kravtsov et al. 2004; Conroy, Wechsler \& Kravtsov 2006; Conroy \& Wechsler 2009; Moster et al. 2010; Moster, Naab \& White 2013; Behroozi, Wechsler \& Conroy 2013; Reddick et al. 2013). In its simplest form, abundance matching places galaxies within haloes based on their relative ranking: the most massive galaxy goes in the most massive halo and on down the rank-ordered lists of galaxies and haloes. The

^E-mail: jeremy.tinker@nyu.edu success of abundance matching suggests a null hypothesis that galaxy properties only care about the mass of their host halo. However, correlations between galaxy and other halo properties at fixed halo mass could manifest in spatial clustering; this is the well-known assembly bias effect, in which haloes of fixed mass cluster differently depending on their formation history and internal structure (Gao, Springel \& White 2005; Wechsler et al. 2006; Gao \& White 2007; Wetzel et al. 2007; Li, Mo \& Gao 2008; Dalal et al. 2008). This idea has been tested in various contexts but with conflicting results.

Using myriad galaxy clustering statistics, a number of studies found no evidence for correlations of galaxy properties with environment at fixed halo mass (Abbas \& Sheth 2006; Skibba et al. 2006; Tinker et al. 2008). In contrast, many results using 
galaxy group catalogues to identify dark matter haloes show that galaxy properties depend on halo formation history as well as mass (Yang, Mo \& van den Bosch 2006; Wang et al. 2008, 2013; Lacerna, Padilla \& Stasyszyn 2014), provided there are no biases in the halo masses induced by the group finding method (Campbell et al. 2015). Kauffmann et al. (2013) find that star formation rates of galaxies in separate haloes are correlated, an effect known as 'galactic conformity'. To model the conformity results, Hearin \& Watson (2013) presented the 'age-matching' model. In this model, galaxies are first connected to haloes via abundance matching. Galaxies in bins of stellar mass are rank ordered by the formation time of their haloes and then galaxy colour (or star formation rate) is imparted on this rank-ordered list. This process matches both the observed stellar mass function and the observed distribution of colours at all $M_{*}$. Thus the oldest haloes contain the reddest galaxies, while to the youngest haloes contain the bluest galaxies. A complication of interpreting the age-matching model in the context of galaxy formation is that 'age' is difficult to define objectively ( $\mathrm{Li}$ et al. 2008), and the redshifts at which haloes accrue most of their mass may not correlate (or may anticorrelate) with the redshifts at which galaxies form or accrete most of their stars. Additionally, Geha et al. (2012) find a limiting stellar mass of $10^{9} \mathrm{M}_{\odot} h^{-2}$ for field galaxies, below which no galaxies are quenched. This represents a threshold below which halo formation history can, by definition, play no role in whether a galaxy is quenched because there are none, even though the amplitude of the assembly bias effect usually gets stronger as halo mass gets smaller (Gao et al. 2005; Wechsler et al. 2006; Gao \& White 2007), depending on which secondary halo property is used.

In this series of papers, we test the assumption that halo growth and galaxy growth are correlated. We construct this test in several distinct regimes. In this paper, we focus on whether halo growth rate correlates with whether a galaxy is quenched of its star formation and resides on the red sequence. We use the spectral diagnostic $\mathrm{D}_{n} 4000$ to separate galaxies into star-forming and quiescent samples. In a companion paper, we test whether halo growth rate - as well as other galaxy properties - correlates with galaxy star formation rate within the star-forming main sequence. Finally, this series will also present new measurements of galactic conformity in the local universe. To perform these tests, we use group catalogues created from the NYU Value-Added Galaxy Catalog (Blanton et al. 2005), which in turn were created from data from Data Release 7 of the Sloan Digital Sky Survey (SDSS; York et al. 2000; Abazajian et al. 2009). Results from the group catalogues are compared to models created with high-resolution cosmological $N$-body simulations. We will focus exclusively on 'central' galaxies - galaxies that reside at the centre of distinct haloes, not orbiting within the virial radius of a larger halo. The latter we classify as satellite galaxies. The formation histories of central and satellite galaxies are quite different and are acted upon by different physical mechanisms (see e.g. Wetzel et al. 2013 and citations within). To isolate the effect of halo assembly bias on the galaxy population, focusing on central galaxies makes this comparison clearer.

Throughout, we define a galaxy group as any set of galaxies that occupy a common dark matter halo, and we define a halo as having a mean interior density 200 times the background matter density. A host halo is a halo that is distinct: its centre does not reside within the radius of a larger halo. We will use the terms halo and host halo interchangeably in this work. A subhalo is one whose centre is located within the radius of a larger halo. For all distance calculations and group catalogues we assume a flat, $\Lambda$ cold dark matter $(\mathrm{CDM})$ cosmology of $\left(\Omega_{m}, \sigma 8, \Omega_{b}, n_{s}, h_{0}\right)=(0.27$, $0.82,0.045,0.95,0.7)$. Stellar masses are in units of $\mathrm{M}_{\odot} h^{-2}$. We will sometimes refer to galaxies as 'blue' and 'red' to refer to their intrinsic star formation; 'red' means red-and-dead rather than red by dust contamination.

\section{DATA, MEASUREMENTS AND METHODS}

\subsection{NYU Value-Added Galaxy Catalog}

To construct our galaxy samples, we use the NYU Value-Added Galaxy Catalog (VAGC; Blanton et al. 2005) based on the spectroscopic sample in Data Release 7 (DR7) of the SDSS (Abazajian et al. 2009). We construct four volume-limited samples that contain all galaxies brighter than $M_{r}-5 \log h=-18,-18.5,-19$ and -20 , respectively. Within each volume-limited sample, we determine the stellar mass at which the sample is complete. The stellar masses are also taken from the VAGC and are derived from the KCORRECT code of Blanton \& Roweis (2007), which assumes a Chabrier (2003) initial mass function. In order of increasing luminosity, the stellar mass samples are complete at $\log M_{*}=9.4,9.6,9.8$ and 10.3, where stellar mass are once again in units of $\mathrm{M}_{\odot} h^{-2}$ (see Fig. 1 in Tinker, Wetzel \& Conroy 2011). After creation of a volume-limited sample in $r$-band magnitude, the stellar mass at which all galaxies more massive than this value are in the volume. This process usually reduces the size of the sample by $\sim 1 / 3$ relative to the $r$-band volume-limited sample.

For galaxy pairs that are too close to obtain spectra because of the 55 arcsec width of SDSS fibers ('fibre collisions'), we use the internal correction to the fibre corrections within the VAGC, namely that the collided object is given the redshift of the nearest galaxy in terms of angular separation, provided that this redshift is in agreement with the photometric redshift obtained by with the SDSS photometry (Blanton et al. 2005).

Using galaxy colour as a proxy for star formation activity can be problematic, as dust reddening can cause a gas-rich disc galaxy to be classified as a red sequence object (Maller et al. 2009; Masters et al. 2010). To avoid this problem, we use $D_{n} 4000$, which is a diagnostic of the light-weighted age of the stellar population and thus is sensitive to the integrated star formation history of the galaxy. We obtain these quantities from the JHU-MPA spectral reductions ${ }^{1}$ (Brinchmann et al. 2004). We use $\mathrm{D}_{n} 4000<1.6$ as our proxy for classifying a galaxy as quenched. This value roughly corresponds to the minimum in the bimodal distribution of $\mathrm{D}_{n} 4000$ for central galaxies. We find that the quenched fraction is the same within the errors when using a specific star formation rate (sSFR) cut at $\log \mathrm{sSFR}=-11$, and a cut in equivalent width of $\mathrm{H}_{\delta}=0 \AA$.

\subsection{Measuring large-scale environment}

For each galaxy, we estimate the large-scale environment by counting the number of neighbouring galaxies within a sphere of radius $10 h^{-1} \mathrm{Mpc}$ centred on each galaxy. This quantity is a biased indicator of the dark matter density field, but at $10 h^{-1} \mathrm{Mpc}$ this bias is a simple linear factor and any stochasticity is minimal. The scale dependence of halo bias largely goes away at this scale (Tinker et al. 2005). We count the number of galaxies above the corresponding magnitude threshold for the each sample, and so the tracer of the density field has a different bias for each sample. We do not correct for this between the samples, but note that the relative bias between the different samples is at the $\sim 5$ per cent level (Swanson

\footnotetext{
${ }^{1}$ http://www.mpa-garching.mpg.de/SDSS/DR7/
} 


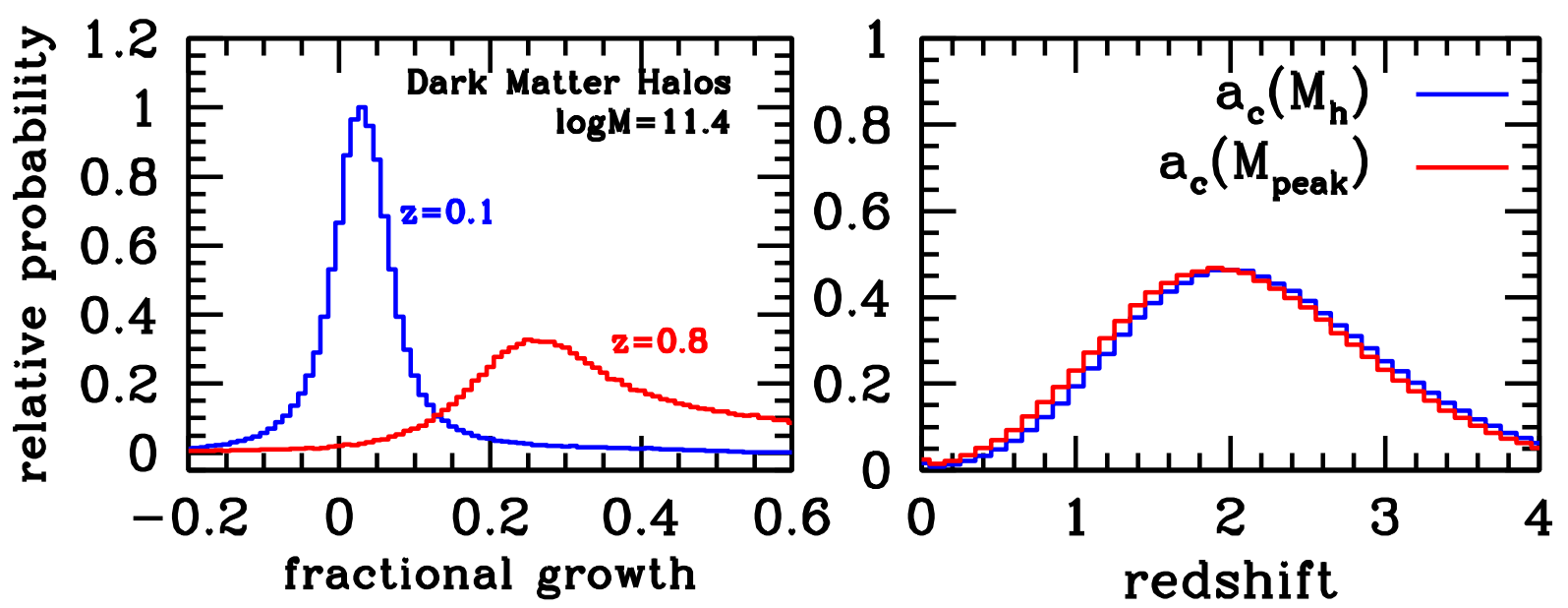

Figure 1. Left-hand panel: fractional growth of haloes from $z=0.1$ to 0 (blue histogram) and $z=0.8$ to 0 (red histogram) for haloes of $\log M_{\mathrm{h}}=11.4$. Over the short timeframe of $z=0.1 \rightarrow 0$, a significant fraction of haloes exhibit negative growth $(\sim 20$ per cent). This fraction is negligible for the $z=0.8 \rightarrow$ 0 timeframe. Right-hand panel: the distribution of halo formation epochs, as defined by the $a_{c}$ parameter of Wechsler et al. (2002). The blue histogram shows the standard result when using halo mass as a function of time $\left(M_{\mathrm{h}}\right)$. The red histogram shows the result when using $M_{\text {peak }}(z)$ to determine halo growth. See the text for details.

et al. 2008). This galaxy density measurement is affected by galaxy peculiar velocities, but this effect is minimal at $10 h^{-1} \mathrm{Mpc}$, as we demonstrate in appendix A in Tinker et al. (2011). We also choose $10 \mathrm{~h}^{-1} \mathrm{Mpc}$ because it represents a clear distinction from a galaxy's small-scale environment as encapsulated by its host halo. The average dark matter mass on this scale is $\sim 4 \times 10^{14} h^{-1} \mathrm{M}_{\odot}$, thus the dark matter haloes even for rich groups are still $\sim 10$ percent of the total mass on that scale, and less if the region is above the mean density. In tests we find that our results show little dependence on the exact smoothing scale chosen. The mean number of galaxies per $10 \mathrm{~h}^{-1} \mathrm{Mpc}$ sphere is $103,72,51$ and 21 galaxies for our volume-limited samples, going from faint to bright.

To correct for survey geometry and incompleteness, we use random catalogues. For each volume-limited sample, we produce a catalogue of $10^{7}$ random points distributed with the angular selection function of SDSS DR7 using the angular mask provided with the VAGC in combination with the software package MANGLE (Swanson et al. 2008). Each random point is also assigned a random redshift such that the comoving space density of randoms is constant with redshift. For each galaxy, we correct for incompleteness by multiplying the observed number of galaxies by the ratio of the number of random points divided by the expected number of randoms if the completeness were unity. The large number of random points ensures that shot noise within each $10 \mathrm{~h}^{-1} \mathrm{Mpc}$ sphere is at the sub-percent level. We restrict ourselves to only using spheres with at least 50 per cent completeness. However, in tests we find a negligible difference when using all spheres, or when increasing the limit to 90 percent. Above this level, we impart noise in the calculation by reducing the number of available spheres.

\subsection{Group finding algorithm}

We use the halo-based group-finding algorithm presented in Tinker et al. (2011), which is, in turn, based on the algorithm of Yang et al. (2005). In brief, the group finder uses the abundance matching ansatz to assign halo masses to groups, iterated until convergence. The resulting group catalogue is a robust decomposition of the entire galaxy population into central galaxies and satellite galaxies. This group finder has been thoroughly vetted in Tinker et al. (2011) as well as Campbell et al. (2015), which specifically investigated colour-dependent statistics derived from the group finders. Campbell et al. (2015) concluded that our group finder can robustly identify red and blue centrals and satellites as a function of their stellar mass, but the assignment of halo masses can be biased when splitting the sample by colour, thus biasing the measurement of $f_{\mathrm{Q}}$ as a function of halo mass. Thus when using the group catalogue, we will only divide the results based on $M_{*}$ and not $M_{\mathrm{h}}$. Fig. 3 shows the relationship between central galaxy stellar mass and halo mass as derived from our three volume-limited samples. The agreement between the three samples, as well as the agreement with other abundance matching and clustering models, shown in the Figure, demonstrate that the halo masses of the overall sample - without splitting into quenched and star-forming subsamples - is reasonable.

\subsection{Numerical simulations and defining halo growth}

We compare the measurements of $f_{\mathrm{Q}}$ as a function of $\delta$ from the group catalogue to expectations from dark matter haloes. We use the 'Chinchilla' simulation (Becker et. al. in preparation), run using a variant of the GADGET- 2 cosmological $N$-body code (Springel 2005) known as L-GADGET2. The box size is $400 h^{-1} \mathrm{Mpc}$ per side, evolving a density field resolved with $2048^{3}$ particles, yielding a mass resolution of $5.91 \times 10^{8} h^{-1} \mathrm{M}_{\odot}$. The cosmology of the simulation is flat $\Lambda \mathrm{CDM}$ consistent with recent CMB results, with $\Omega_{m}=0.286$, $\sigma_{8}=0.82, h=0.7$, and $n_{s}=0.96$. This is slightly higher matter density than assumed for the group catalogues, but the change makes negligible difference in any comparison.

Haloes are found in the simulation using the ROCKSTAR code of Behroozi, Wechsler \& Wu (2013). Halo masses are defined as spherical overdensity masses according to the radius at which their internal mean density is 200 times the background density. We use Consistent Trees (Behroozi et al. 2013) to track the merger and growth history of each halo in the simulation, and we use these histories to determine the growth rate of each halo.

Fig. 1 shows the fractional growth of dark matter haloes at low mass over different redshift baselines. We note that we are only showing results for haloes that are identified as host haloes (no subhaloes) at $z=0$. We define fractional growth as $1-M(z) / M_{0}$, where $M_{0}$ is the present-day halo mass and $M(z)$ is the mass at an 
earlier epoch. Haloes of this mass are likely to contain galaxies of $\log M_{*}=9.4$ (cf. Fig. 3). Detecting assembly bias in survey data has a particular challenge: assembly bias usually increases with decreasing halo mass, but smaller mass haloes contain dimmer galaxies that can only be seen in small volumes. The choice of $\log M_{\mathrm{h}}=11.4$ represents a compromise between these two effects: halo assembly bias seen in simulations is significant, but SDSS still probes these galaxies at cosmological volumes.

Over the redshift baseline $z=0.8 \rightarrow 0$, haloes of this mass scale grow on average $\sim 30$ per cent, but with a wide distribution. Notably, the fraction of haloes with negative growth over this timeframe is negligibly small. Over the timeframe of $z=0.1 \rightarrow 0$, the variance in halo growth rates is much smaller, but roughly a quarter of haloes loses some mass. Some of this is noise, ${ }^{2}$ but these haloes exhibit the strong clustering indicative of assembly bias (as we will see in Section 3), indicating that noise is a minority contributor. Hereafter, we will refer to these redshift baselines as ' $\Delta z$ '. For $\Delta z=0.8$, this definition is over an intermediate timespan $(\sim 7 \mathrm{Gyr})$ over which most of these quenched central galaxies arrive on the red sequence. This result, from Tinker et al. (2013), drives our choice of $\Delta z=0.8$. For $\Delta z=0.1$, this definition is a measure of short-term growth of the dark matter haloes. To implement the age-matching model using these definitions, haloes are rank ordered from lowest to highest fractional growth in bins of halo mass. The lowest are considered the oldest, while the haloes that have grown the most over that timespan are considered the youngest.

In the right-hand panel, we show the 'age' of the same haloes as defined by the $a_{c}$ parameter from Wechsler et al. (2002), which identifies the epoch where halo growth changes from rapid accretion to slower growth. This is qualitatively similar to using halfmass epoch, defined as the redshift at which a halo reached half its present-day value, although the median of $z\left(M_{1 / 2}\right)$ is lower than that of the median redshift of $a_{c}$. When we quantify assembly bias of haloes, we find little difference between using $z\left(M_{1 / 2}\right)$ and $a_{c}$, thus we will focus on $a_{c}$. The two histograms in this panel show $a_{c}$ for two different definitions of halo mass: (1) the current halo mass at any time, $M_{\mathrm{h}}(z)$; and (2) the peak halo mass up to that time $M_{\text {peak }}(z)$. As shown in the left-hand panel, over short time intervals there can be significant dark matter mass loss. In fact, small haloes can be accreted on to a larger halo but have too much kinetic energy to remain within the larger halo, eventually exiting the larger halo after one pericentric passage. These are called 'splashback' haloes, and this process can lead to significant stripping of the dark matter halo. $M_{\text {peak }}(z)$ is a monotonically increasing function, thus for haloes that experience tidal stripping, $M_{\mathrm{h}}(z)$ will be smaller than $M_{\text {peak }}(z)$. This has a small but visible impact on the distribution of formation epochs, pushing $a_{c}$ to slightly smaller redshift. When rank-ordering haloes by their age (or fractional growth), haloes that have experienced significant tidal encounters get pushed to the top of the list. Thus, in the age-matching model of Hearin \& Watson (2013), these haloes house the oldest galaxies. Using $M_{\text {peak }}$ to calculate $a_{c}, z_{1 / 2}$, or halo growth significantly reduces the impact of tidal events or splashback galaxies on the ordering of the list. The overall effect

\footnotetext{
${ }^{2}$ We estimate the noise in assigning halo masses in the ROCKSTAR code by calculating the snapshot-to-snapshot variance around the mean trend in halo growth for each halo for the five snapshots that cover the redshift range $z=0.1 \rightarrow 0$. The variance depends on the order of the polynomial used to fit for the mean trend in $M_{\mathrm{h}}(z)$, but for a second-order polynomial the variance is 1.3 per cent. We conclude that this is an upper limit on the noise in estimating halo mass. The variance in the fractional growth is 4 per cent. Assuming Gaussian statistics, removing the contribution from noise would reduce this only to 3.6 per cent.
}

on the distribution of halo ages is small, but as we will see in the following section, this choice has a major impact on the predicted strength of assembly bias.

\subsection{Comparing simulations to observations}

To compare simulation results to galaxy results binned as a function of environment, we measure the density around each halo in the simulation in the same manner as for the galaxies. This means that we need to create mock samples of galaxies matching the $r$-band samples used to measure local galaxy density around the central galaxies from the group catalogue. Using the halo occupation distribution (HOD) fitting results of Zehavi et al. (2011) from the SDSS main galaxy sample, we populate the simulation with galaxies that match the density and clustering of each of our volume-limited samples. Using the distant-observer approximation and the $z$-axis of the box as the line of sight, the top-hat redshift-space galaxy densities are measured around each halo, in a similar manner as was done for central galaxies in the SDSS group catalogue.

Once this is accomplished, our method of comparing theoretical age-matching models to data is as follows: we use the stellar mass to halo mass relation of Fig. 3 to select the haloes with which to compare a given stellar mass bin. ${ }^{3}$ Once those haloes are selected, within each halo mass bin we rank order the haloes by their age or growth property. In the full age-matching model, the observed values of $\mathrm{D}_{n} 4000$ would be abundance-matched on to the haloes, with the oldest - or slowest growing - halo having the highest $\mathrm{D}_{n} 4000$ value, and so on down the rank-ordered lists. If, for example, 20 per cent of observed galaxies have $\mathrm{D}_{n} 4000<1.6$, then the 20 per cent oldest haloes would also have $\mathrm{D}_{n} 4000<1.6$, and thus the 20 per cent oldest haloes would be classified as quenched in this model. Here, we skip the middle man and utilize the observed quenched fraction to set the break point in halo age or growth between haloes that contain quenched and star-forming central galaxies. Once we know which haloes are classified as quenched, we calculate how the quenched fraction varies as a function of environment using the mock galaxies described above. We note that, although the group finding algorithm can impart significant biases on $f_{\mathrm{Q}}^{\text {cen }}$ as a function of $M_{\mathrm{h}}$, these biases do not emerge when forward modelling the haloes to compare to $f_{\mathrm{Q}}^{\text {cen }}$ as a function of stellar mass. This is described further in Appendix B.

In an appendix we show the results of two additional proxies for halo age: the redshift at which a halo reaches half its presentday mass, $z_{1 / 2}$; and halo concentration, $c_{\mathrm{vir}}$, which has been shown to correlate tightly with formation history (Wechsler et al. 2002) and is one of the primary quantities through which halo assembly bias manifests. We do not include these in the main text as they are quantitatively similar to the definitions already in hand. In this appendix, we also show the 'break points' delineating old and young haloes.

\section{RESULTS}

\subsection{Central-satellite decomposition of the SDSS}

Fig. 2 shows an example of the group finder applied to one of our volume-limited samples. The top panel shows galaxies in the

\footnotetext{
${ }^{3}$ We only use the HOD results of Zehavi et al. (2011) because they yield halo occupation of threshold samples defined by luminosity, whereas our comparison of age-matching models focuses on narrow bins of galaxy stellar mass
} 

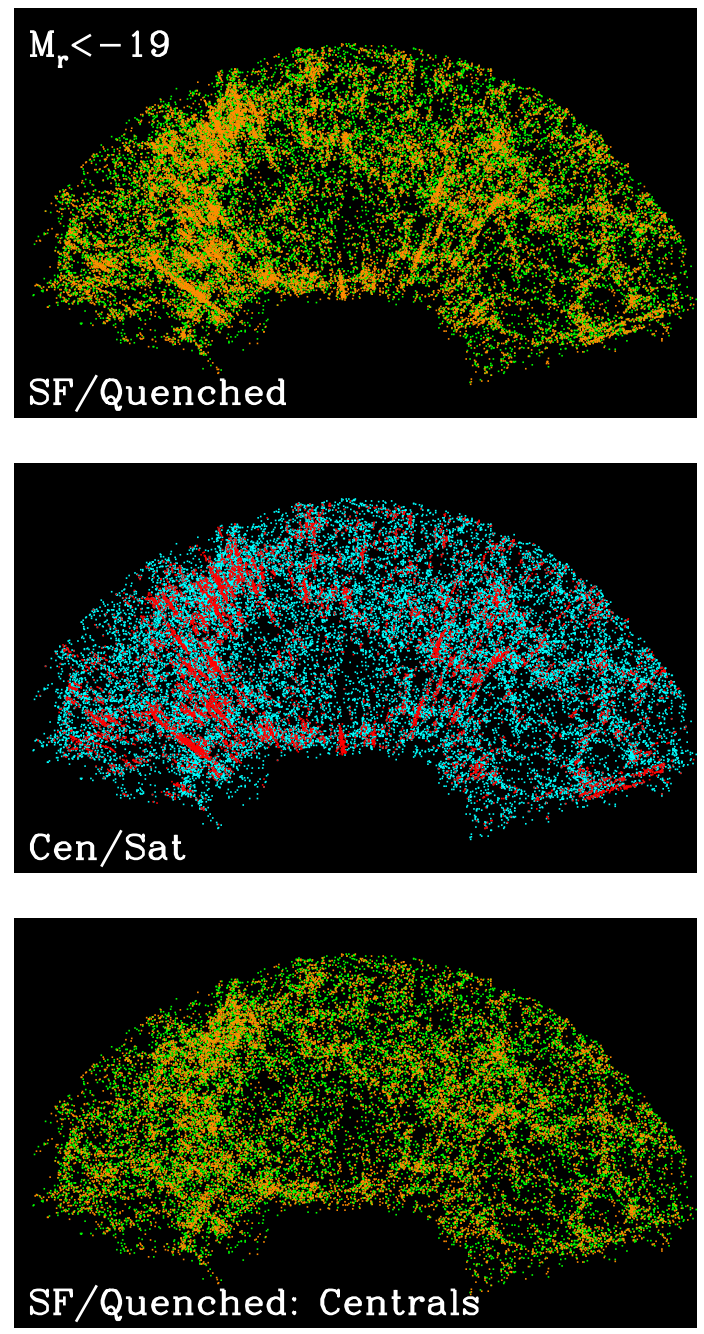

Figure 2. Upper panel: a slice through a volume-limited sample of SDSS galaxies with $M_{r}<-19$. This sample extends to $z=0.064$. The colour types of the points correspond to their star formation activity: the green points are on the star-forming main sequence while the orange points are quiescent galaxies on the red sequence. Middle panel: the same set of galaxies, now categorized as central galaxies and satellite galaxies by the group finder. The group finder clearly identifies the fingers of God as redshifted galaxy groups, but some satellites exist in lower density environments. Lower panel: the same as the upper panel, but now only central galaxies are being plotted. With the satellites removed, the finger-of-God effect is no longer present, but there is a substantial fraction of quiescent galaxies, many of which reside in underdensities and voids.

North Galactic Cap footprint (only plotting galaxies with $\delta<22^{\circ}$ to avoid crowding), where the colour of the point indicates whether the galaxy is star-forming or quiescent. The middle panel shows the same galaxies, but now colour indicates whether the galaxy is a central or a satellite. The group catalogue clearly identifies the 'fingers of God' created by the large virial motions of satellite galaxies. Satellites are mostly found in high-density regions along filaments in the cosmic web, although some satellites are still found occasionally out in the field. The bottom panel shows the starforming and quiescent breakdown of the sample, but now for central galaxies only. Although satellites are more likely to be on the red sequence than central galaxies, this panel elucidates two aspects of central galaxies: (1) the overall fraction of central galaxies on the red sequence is significant at these masses; and (2) that quenched

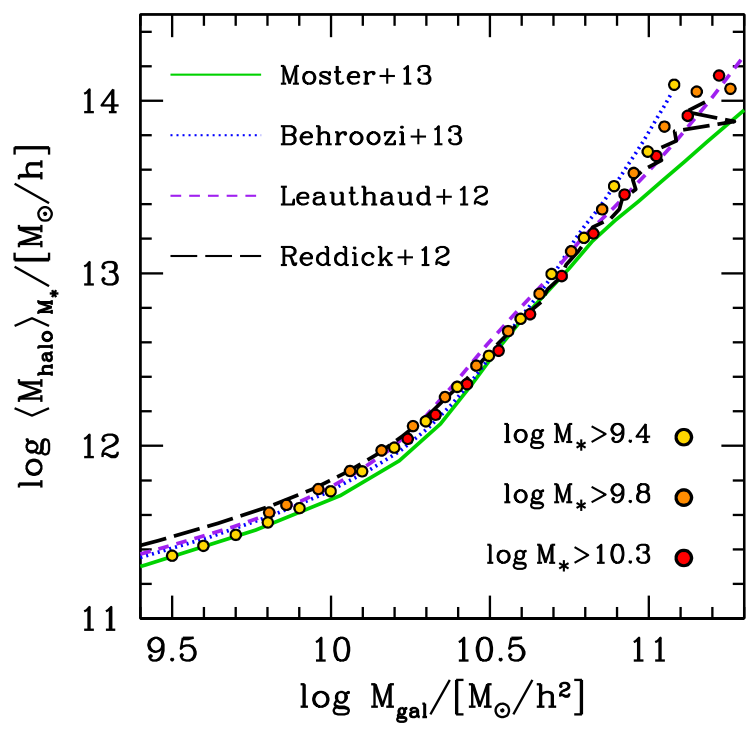

Figure 3. Average halo mass in bins of $\log M_{*}$ for central galaxies from the three volume-limited group catalogues. The four curves represent a sample of stellar to halo mass relation from abundance matching (Behroozi, Wechsler \& Conroy 2013; Moster, Naab \& White 2013) and studies that combine abundance and clustering (Leauthaud et al. 2012; Reddick et al. 2013). We note that the group-finding algorithm is also based on abundance matching of the total group mass on to host halo mass, which reduces to a standard abundance matching result at low central galaxy stellar masses where the number of satellites is small.

central galaxies exist at all densities, even the deepest void in the galaxy distribution.

Fig. 3 shows the relationship between $M_{\mathrm{h}}$ and $M_{*}$ for central galaxies for three volume-limited group catalogues. The results between the catalogues are in excellent agreement with one another, as well as with results from the literature constraining this relationship from different methods. We use this relationship to make subsequent comparisons between our halo catalogues and the SDSS measurements. Although the mean $M_{\mathrm{h}}$ in bins of $M_{*}$ is not equivalent to the inverse relationship, the differences between Fig. 3 and its inverse only appear at $M_{*} \gtrsim 10^{10.6}$, above the limit for our comparisons.

\subsection{Quenched fraction of central galaxies and large-scale density}

The correlation between density and galaxy properties is wellknown (see Blanton \& Moustakas 2009 for a thorough review). Progressing from low-to-high densities, the fraction of galaxies that are red-and-dead, $f_{\mathrm{Q}}$, monotonically grows (see e.g. Oemler 1974; Davis \& Geller 1976; Dressler 1980 for canonical works and Hogg et al. 2004; Kauffmann et al. 2004; Blanton et al. 2005; Baldry et al. 2006; Park et al. 2007; Bamford et al. 2009 for more recent measurements). However, this observation combines central galaxies that live in low-mass dark matter haloes with satellite galaxies that orbit within high-mass dark matter haloes. The observed trend is driven by the density dependence of the halo mass function: more massive haloes live in more dense environments, and in turn have a higher fraction of quenched galaxies. The question we pose here is, when restricting the sample to central galaxies of fixed stellar mass, which is a reasonable proxy for fixed halo mass, what is the correlation between $f_{Q}$ and environment? 

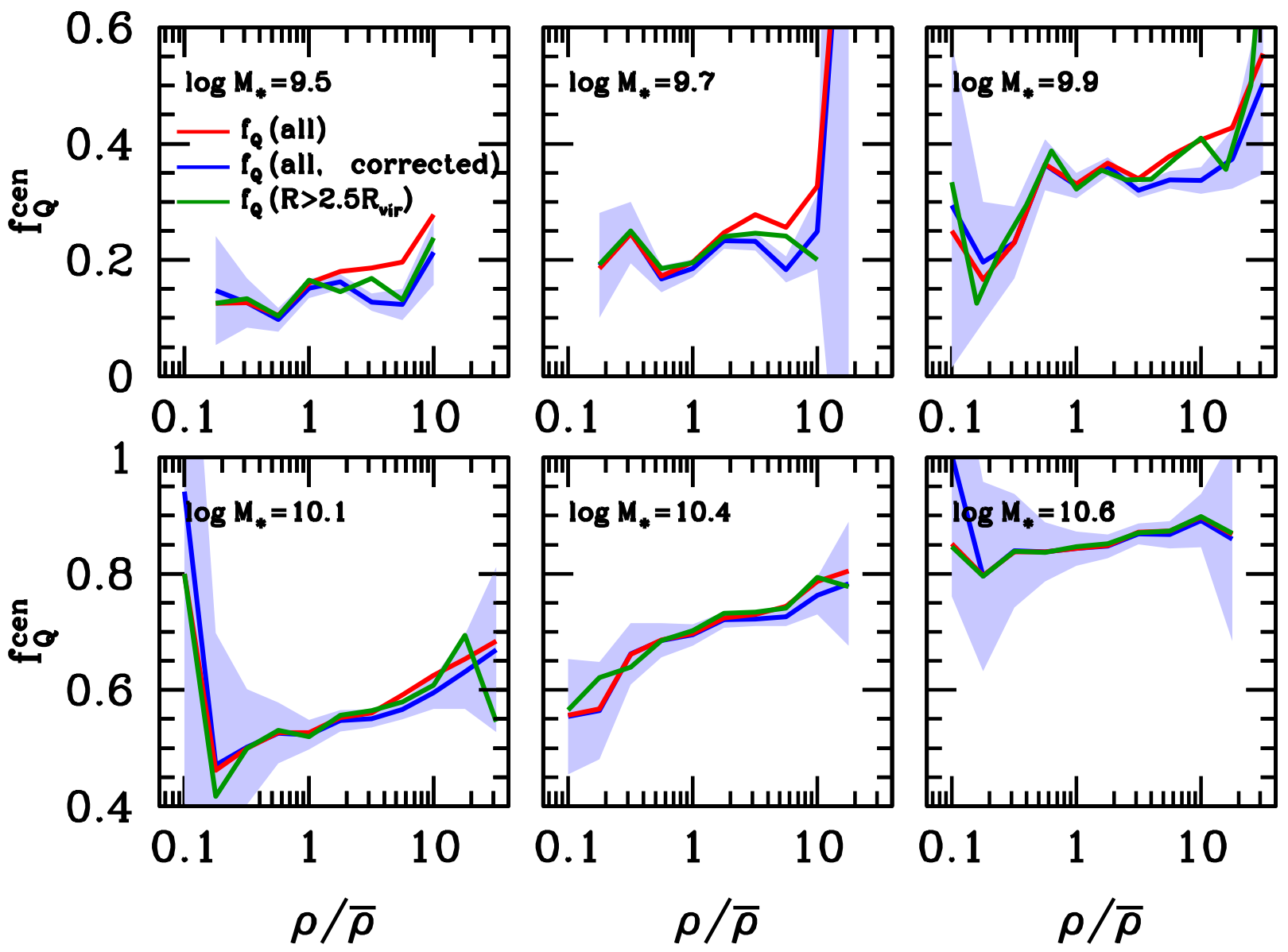

Figure 4. Measurements of the quenched fraction of central galaxies, $f_{\mathrm{Q}}^{\text {cen }}$, as a function of large-scale density. Each panel shows three methods for measuring $f_{\mathrm{Q}}^{\text {cen }}$ : (1) the raw measurement that uses all centrals in the group catalogue; (2) the measurement using all centrals in the group catalogue, but statistically corrected for misidentification of centrals and satellites in the group-finding process; and (3) raw measurement that excludes centrals that are within $2.5 R_{\mathrm{vir}}$ of a larger group. The shaded region around the corrected measurement is the error in the mean, and is representative of the error on the other two measurements. At $\rho \lesssim 1$, where the abundance of massive groups is low, all three measurements are essentially the same. At high densities and low stellar masses, the measurements separate at high densities. For high stellar mass bins, the frequency of groups more massive than the host haloes being probed is small, and all three measurements are consistent at all $\rho$.

We define red-and-dead galaxies as those with $\mathrm{D}_{n} 4000>1.6$. We find that this value of 1.6 faithfully follows the minimum of the distribution of $\mathrm{D}_{n} 4000$ values between the star forming main sequence and the red sequence, irrespective of galaxy stellar mass. We also note that the results are nearly indistinguishable when using SSFR as our indicator of quiescence. Fig. 4 shows the quenched fraction of central galaxies, $f_{\mathrm{Q}}^{\text {cen }}$, as a function of large-scale galaxy density, measured in three different ways:

(1) We present the raw measurements of $f_{\mathrm{Q}}^{\mathrm{cen}}(\rho)$ in which all central galaxies in the group catalogue are used.

(2) We apply a statistical correction to $f_{\mathrm{Q}}^{\text {cen }}(\rho)$ to remove biases imparted by the group-finding process.

(3) We measure $f_{\mathrm{Q}}^{\text {cen }}(\rho)$ after removing all central galaxies that are within $2.5 R_{\text {vir }}$ of a larger group, defined as a group with a larger halo mass.

For (2), $f_{\mathrm{Q}}^{\text {cen }}$ is corrected for impurities in the group catalogue as in appendix $\mathrm{C}$ in Tinker et al. (2011). In the group-finding process, centrals and satellites are sometimes misclassified, leading to $\sim 10$ per cent of central galaxies in the catalogue being true satellites. This effect increases $f_{\mathrm{Q}}^{\text {cen }}$ because satellite galaxies always have higher quenched fractions at fixed $M_{*}$, and the misclassification occurs more frequently in higher density regions that contain more satellites. The statistical correction described in Tinker et al. (2011) is applied directly to measurements of $f_{\mathrm{Q}}$, and robustly accounts for biases in the group-finding process. For $M_{*}<10^{10} \mathrm{M}_{\odot} h^{-2}$, the corrected and uncorrected measurements of $f_{\mathrm{Q}}^{\text {cen }}$ at low densities are the same. This is expected because the abundance of satellite galaxies is negligible at $\rho<1$. At higher $\rho$, the correction factor lowers $f_{\mathrm{Q}}$ by roughly 0.05 to 0.10 , also expected from the amount of misclassification in the group catalogue (appendix $\mathrm{C}$ in Tinker et al. 2011). At higher stellar masses, the corrected and uncorrected results are consistent at all $\rho$ due to the fact that there is a smaller difference in the quenched fractions of central and satellite galaxies than for lower $M_{*}$.

For (3), as discussed in Section 2.4, halo growth can be negative. How this impacts the growth of galaxies is not fully understood, but splashback galaxies are subject to environmental processes, such as ram pressure and strong tidal stripping that more isolated galaxies are not subjected to. Thus they are not clean tests of the correlation of halo growth history to galaxy formation and it makes sense to treat them as a separate class of galaxies. Wetzel et al. (2014) showed that splashback galaxies essentially behave the same as satellite galaxies, meaning that after the initial accretion event 

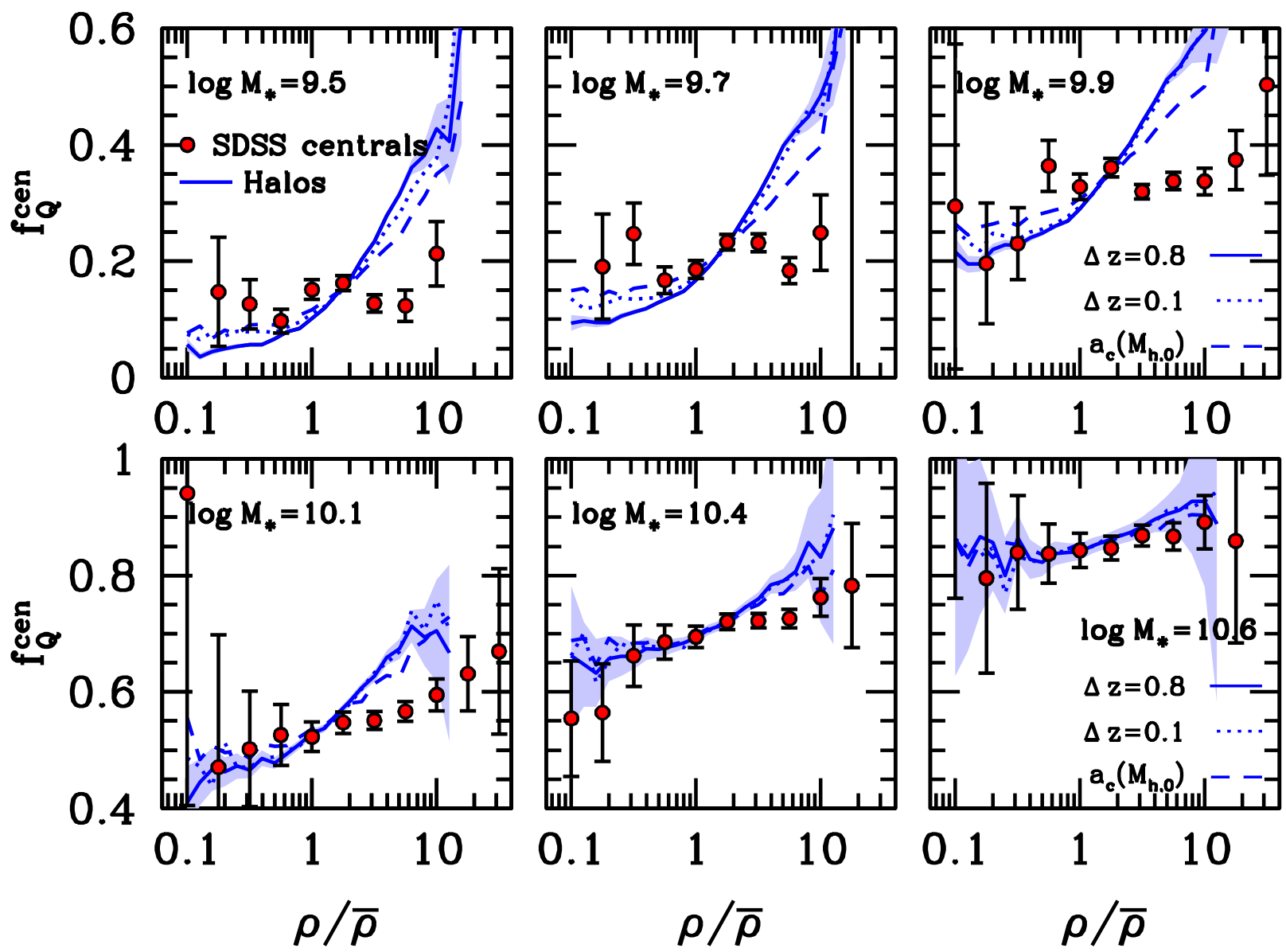

Figure 5. The quenched fraction of central galaxies, $f_{\mathrm{Q}}^{\text {cen }}$, as a function of large-scale galaxy density, for six bins in $M_{*}$. Galaxy density measurements are described in Section 2.2. Error bars are the error in the mean in each $\rho$ bin. In each panel, we compare these measurements to expectations from the age-matching model, which stipulates that redder galaxies (or, in these data, galaxies with the largest values of $\mathrm{D}_{n} 4000$, which implies that they have the oldest stellar populations) live in older haloes. We use the group catalogue to estimate the halo masses for each bin in stellar mass. In each bin in halo mass, the haloes are rank ordered by three different definitions of age: (1) their fractional growth since $z=0.8$; (2) their fractional growth since $z=0.1$; and (3) their formation epoch as defined by $a_{c}\left(M_{\mathrm{h}}\right)$. We set the break point between 'old' and 'young' haloes to match the value of $f_{\mathrm{Q}}^{\text {cen }}$ in each bin. As expected from halo assembly bias, the old fraction of haloes depends strongly on large-scale environment. The assembly bias gets less strong monotonically with increasing $M_{*}$. The data, in contrast, show the opposite trend. At low stellar masses, there is little to no dependence of $f_{\mathrm{Q}}$ on environment. As $M_{*}$ increases, $f_{\mathrm{Q}}$ shows a positive trend with $\rho$. This figure is an updated version of the one presented in Tinker et al. (2011), with new simulation predictions and correcting for an error in the density calculations around the galaxies.

their evolution is unchanged for a long delay time and then they rapidly quench their star formation. The key quantity is the time of the initial accretion event, regardless of the previous evolutionary history of the halo; i.e. regardless of whether it was an early-forming halo or late-forming halo before the accretion event. This then begs the question: if splashback galaxies and haloes are removed from consideration, what are the observations and theoretical predictions?

To implement (3), we remove all central galaxies with projected separation $R<2.5 R_{\mathrm{vir}}$ of a larger group and $\Delta v<1000 \mathrm{~km} \mathrm{~s}^{-1}$ with respect to the central galaxy of the larger group. These choices are motivated by the results of Wetzel et al. (2014) and references therein. The measurements of $f_{\mathrm{Q}}^{\text {cen }}$ after this process split the difference between the raw measurements and the corrected measurements. The statistical correction is not applied here because it is only applicable on the full sample of galaxies. But in comparison to the raw data using all centrals, removing galaxies near groups lowers $f_{\mathrm{Q}}$ in high densities and low stellar masses. For $M_{*} \gtrsim 10^{9.8}$ there is little difference between the raw measurements and those with no galaxies near groups.
Regardless of how $f_{\mathrm{Q}}^{\text {cen }}$ is measured, the results indicate that, at $M_{*}<10^{10} \mathrm{M}_{\odot} h^{-2}$, there is little correlation between quenched fraction and large-scale environment. At $M_{*}>10^{10} \mathrm{M}_{\odot} h^{-2}$, there is a shallow but significant positive slope of $f_{\mathrm{Q}}^{\text {cen }}$. We will compare these results to predictions of the age-matching model in the following sections.

3.3 Does halo growth correlate with a galaxy being on the red sequence?

Fig. 5 shows the measurements of $f_{\mathrm{Q}}^{\text {cen }}$, using all central galaxies and corrected for group-finding biases. The curves show the prediction of the age-matching model, which puts the oldest galaxies into the oldest haloes (once fixing halo mass). We choose $M_{\mathrm{h}}$ by the halo mass assigned to galaxies in each bin, but we note that the halo predictions vary weakly with halo mass (as can be seen in the figure). Thus, minor biases in $M_{\mathrm{h}}$ and scatter between halo mass and stellar mass are not likely to change the predictions. Haloes are rank ordered by three of the metrics for halo age presented in Section 2.4: 

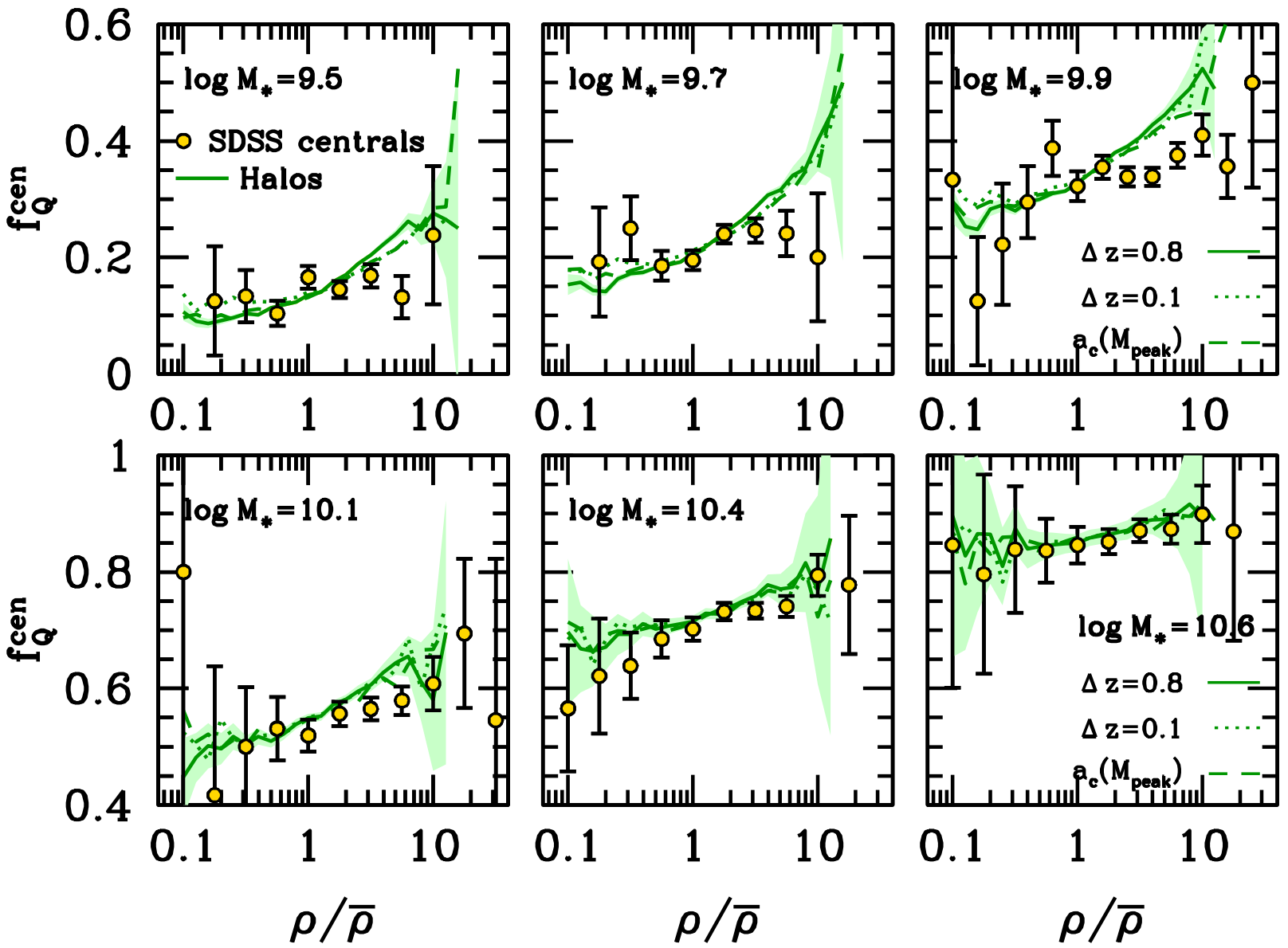

Figure 6. Analogous to Fig. 5, but removing galaxies that are near larger haloes to eliminate the possible effects of splashback encounters. For the data, we remove from the sample any central galaxy that is within $2.5 R_{\mathrm{vir}}$ of a larger halo. The data look nearly the same as in Fig. 5, but recall that those data have been statistically corrected for bias in $f_{\mathrm{Q}}$, while these data have not been corrected in any way. The three curves show the predictions of the old fraction of dark matter haloes. We use the same three definitions of halo age as before. However, for $\Delta z=0.8$ and $\Delta z=0.1$, we remove all haloes that are within $2.5 R_{\text {vir }}$ of a larger halo, just as with the data. For formation epoch, $a_{c}$, we use $M_{\text {peak }}$ rather than $M_{\mathrm{h}}$, but use all haloes in the sample. This removes the impact of tidal events and splashbacks without removing any haloes.

halo growth over the redshift ranges $\Delta z=0.8$ and $\Delta z=0.1$, and the formation epoch $a_{c}\left(M_{\mathrm{h}}\right)$. We set the break point between 'old' and 'young' haloes such that the old fraction of haloes matches the observed $f_{\mathrm{Q}}^{\text {cen }}$ in the data.

The age-matching curves in Fig. 5 indicate how the fraction of old haloes depends on $\rho$. Under the age-matching hypothesis - regardless of age definition - the old fraction has a strong dependence on $\rho$, with the majority of old haloes living in dense environments. This result is consistent with previous results of halo assembly bias, but at odds with the observations at most stellar mass bins, most notably at lower stellar masses. Even at $M_{*}>10^{10} \mathrm{M}_{\odot} h^{-2}$, where there is a measurable trend of $f_{\mathrm{Q}}^{\text {cen }}$ with $\rho$, the standard age-matching model predicts a correlation stronger than that seen in the data. At lower masses, the data and theory are at loggerheads: the strength of the assembly bias is at its largest, but the data show the weakest correlation between $f_{\mathrm{Q}}^{\text {cen }}$ and $\rho$, if at all.

\subsection{Removing the impact of splashback haloes and galaxies}

Fig. 6 shows analogous measurements and models as Fig. 5, only here, splashback effects have been removed. For the SDSS central galaxies, any galaxy that is within a projected separation of $2.5 R_{\mathrm{vir}}$ of a larger halo, along with $\Delta v<1000 \mathrm{~km} \mathrm{~s}^{-1}$, is removed from the sample.

The curves in each panel represent the predictions of the agematching model using the same three definitions of halo age as before. For $\Delta z=0.8$ and $\Delta z=0.1$, the halo samples have been altered in the same fashion as the data: all haloes within $2.5 R_{\mathrm{vir}}$ of a larger halo, using three-dimensional separation, have been removed from the sample. In comparison to the age-matching predictions of Fig. 5, the assembly bias signal is substantially reduced: there is a definite trend of higher old fraction in higher densities, but not nearly as steep as the trend for all haloes. The final age-matching prediction, using $a_{c}$, but now $a_{c}$ is defined using $M_{\text {peak }}$ rather than $M_{\mathrm{h}}$. For this model, no haloes are removed from the catalogue. We include this model here, rather than Fig. 5, to show that the $a_{c}\left(M_{\text {peak }}\right)$ model induces the same level of assembly bias as models that remove all potential splashback effects.

At lower masses, the age-matching model predicts a correlation of $f_{\mathrm{Q}}^{\text {cen }}$ with $\rho$ not seen in the data, although the differences between theory and data are smaller than that seen with the standard agematching implementation. At higher masses, $M_{*}>10^{10} \mathrm{M}_{\odot} h^{-2}$, there is reasonable agreement between the age-matching models and the positive trend of increasing $f_{\mathrm{Q}}^{\mathrm{cen}}$ with $\rho$. 


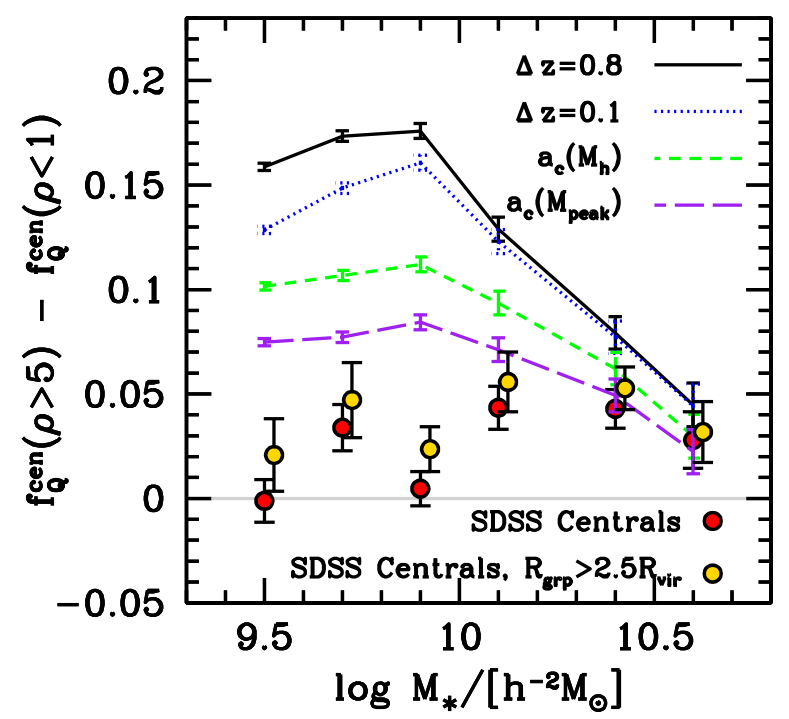

Figure 7. Assembly bias in SDSS central galaxies and in dark matter haloes. The $y$-axis is the difference between the quenched fraction of central galaxies at high densities $(\rho>5)$ and low densities $(\rho<1)$. The red and yellow circles represent SDSS centrals for the full sample (red) and when galaxies near larger groups have been removed (yellow). Recall that the red points have been corrected for biases in the group catalogue. Error bars are error in the mean. Curves show predictions from dark matter haloes for four different definitions of halo age. We do not show any predictions from age-matching models where haloes near groups are removed because they are consistent with the results from the $a_{c}\left(M_{\text {peak }}\right)$ model.

\subsection{Assembly bias in haloes and Galaxies}

Fig. 7 summarizes our results on assembly bias in galaxies and haloes. The $y$-axis shows the difference between the quenched fractions at high and low densities, $f_{\mathrm{Q}}^{\text {cen }}(\rho>5)-f_{\mathrm{Q}}^{\text {cen }}(\rho<1)$, as a function of stellar mass. At low masses, the results are generally consistent with little to no assembly bias. At high masses, there is a statistically robust assembly bias signal, with red fractions being around 0.05 higher at high densities. The overall quenched fraction at these masses approaches unity, thus another way to phrase the result is that the blue fraction of central galaxies in high densities is $10-20$ per cent lower than in lower densities.

The curves show the predictions of the age-matching models for four different age definitions. The first three show the models from Fig. 5, in which all haloes are used at each mass bin. The fourth model uses $a_{c}\left(M_{\text {peak }}\right)$ as the halo age definition, although we note that all the theoretical models from Fig. 6 are consistent with one another. At low stellar mass, Fig. 7 conveys two important points: (1) that the amplitude of the assembly bias signal depends strongly on how one defines halo age; and (2) that none of these models are in particularly good agreement with the data. At high stellar masses, the comparison of haloes and galaxies is quite different. There is still a dependence of the assembly bias signal on age definition, but the prediction of the $a_{c}\left(M_{\text {peak }}\right)$ model is in reasonable agreement with the data. We note that this implies that all models that remove possible splashback haloes will also be in agreement.

\section{DISCUSSION}

The main results of this paper are given below.

(i) The predictions of the age-matching model depend on how age is defined. More specifically, once tidal and splashback effects are removed from consideration by use of $M_{\text {peak }}(z)$ rather than $M_{\mathrm{h}}(z)$, the amount of assembly bias is reduced. This has the largest effect on low-mass haloes $M_{\mathrm{h}} \lesssim 10^{12} h^{-1} \mathrm{M}_{\odot}$.

(ii) At low galaxy mass, $M_{*} \lesssim 10^{10} \mathrm{M}_{\odot} h^{-2}$, the results are consistent with little-to-no assembly bias, implying no relationship between halo age and galaxy quenching for central galaxies.

(iii) At higher galaxy masses, the results are consistent with predictions from the age-matching model after removing the effects of splashback haloes by using $M_{\text {peak }}(z)$ to characterize formation history.

The first point is important for properly framing our expectations from assembly bias and the age-matching model. The extreme assembly bias predictions at low masses are driven by tidal and splashback effects lowering the present-day mass of the halo relative to its peak value at some earlier time. Wetzel et al. (2014) shows that the galaxies within these haloes are not immediately affected by these encounters: after accretion on to a larger halo, the galaxy evolves as though it were still in the field for 3-5 Gyr. Most haloes will be reaccreted by the larger halo during that time, and nearly all will eventually be reaccreted on to the larger halo (or another larger halo nearby). There is a measurable increase in the quenched fraction within a couple virial radii of a larger halo, but splashback galaxies are not a significant contributor to the whole population of central galaxies on the red sequence. Thus standard age-matching predictions generally overestimate the impact of halo formation history on galaxy quenching. One aspect of the age-matching model that has received little attention (and this work is no different) is the possibility of scatter in any halo age-galaxy age correlation. A one-to-one correspondence between these two properties is unlikely and scatter is a key component of the standard abundance matching model. Scatter between halo age and galaxy age would reduce the amplitude the assembly bias in the galaxy population. It is possible that a physically reasonable amount of scatter could reconcile the standard age-matching model with observations at high $M_{*}$. More work is required to define 'physically reasonable scatter', but at low masses the amount of scatter required to bring age-matching into agreement with the data would be so large as to eliminate any effective correlation.

The measurements of $f_{\mathrm{Q}}^{\text {cen }}(\rho)$ cannot be reconciled with the predictions of the age-matching model at low $M_{*}$. This result caps a number of other results that are mutually exclusive with a model that maps halo age on to galaxy age at these mass scales. Tinker et al. (2008) demonstrated that the sizes of voids in red and blue galaxies is consistent with galaxy colour being independent of large-scale environment, and inconsistent with the level of assembly bias seen in red galaxies in, for example, the Croton, Gao \& White (2007) semi-analytic model. When comparing the results of age-matching models to measurements of galaxy clustering and galaxy-galaxy lensing, there are conflicting results in the literature. Hearin et al. (2014) show reasonable agreement between the age-matching model and measurements of colour-dependent clustering and lensing. In contrast, Mandelbaum et al. (2016) and $\mathrm{Zu} \&$ Mandelbaum (2016) find that the predictions of the standard age-matching model are inconsistent with galaxy-galaxy lensing measurements split by colour in bins of galaxy stellar mass. One difference between these two analyses is that Hearin et al. (2014) compare models to data in thresholds of stellar mass, while the other papers compare model and data in bins of stellar mass. Additionally, Zehavi et al. (2011) use a standard halo occupation formalism to fit the colour-dependent clustering of SDSS galaxies in multiple, narrow bins of colour at fixed galaxy luminosity. In the standard HOD 
approach, galaxies occupy haloes based only on the halo mass. Thus, if colour depended significantly on halo age at fixed mass, the standard HOD approach would not be able to fit the clustering data-the prediction of the standard HOD would yield large-scale clustering of red galaxies too low relative to the data. This could be compensated for by making the fraction of red galaxies that are satellites higher, but this would then predict too high clustering at small scales. Zentner, Hearin \& van den Bosch (2014), using mock galaxy samples that contain assembly bias, do obtain a good fit to mock clustering using the standard HOD approach, but the clustering was measured in threshold samples, not magnitude bins as done in Zehavi et al. (2011).

The third point above implies that there is some change in how quenching correlates with halo formation history between low stellar masses and high stellar masses. Either that change manifests from a change in the physical mechanism that quenches galaxies, or that the mechanism is the same but the correlation between that mechanism and halo formation history - i.e. the scatter discussed above - increases significantly as halo mass decreases. Dalal et al. (2008) show that the assembly bias in low- and high-mass haloes, split around $M_{\mathrm{h}} \sim 10^{12} \mathrm{M}_{\odot}$, is caused by different physical mechanisms. As we have noted above, assembly bias in low-mass haloes is driven by tidal encounters and other interactions with the large-scale environment. Assembly bias in high-mass haloes, on the other hand, is imprinted in the primordial density field; early- and late-forming haloes can be identified by the nature of their initial perturbations. The process that quenches low-mass field galaxies must be nearly independent of environment and thus uncorrelated with halo formation history. This does not necessarily imply that all the properties of low-mass field galaxies are uncorrelated with the details of halo growth; star formation rates, galaxy sizes and morphologies may correlate with short term or long term halo growth rates. The results here only indicate that the decision to migrate from the star-forming sequence to the red sequence is not up to the halo, after accounting for halo mass. One caveat is that there may be halo properties that do not correlate with large-scale environment that are coming into play.

In contrast, high-mass galaxies tell a different story. Tinker (2016) shows that, if quenching is induced by a threshold in either galaxy mass or halo mass, the epoch of quenching will depend on halo formation history, with early-forming haloes quenching earlier. Tinker et al. (2012) found that the clustering of X-ray groups depended on the state of the central galaxy; groups with star-forming centrals had higher clustering at $z \sim 1$. The picture these results paint is consistent with the results here; older massive haloes are more likely to contain quenched galaxies than younger haloes, but the overall size of the effect is relatively small compared to the mean quenched fraction of high-mass galaxies.

We will tackle galactic conformity in a future paper in this series, but the results presented here are inconsistent with a model in which assembly bias creates strong large-scale galactic conformity for lowmass galaxies (i.e. conformity outside the virial radii of the haloes in which the galaxies lie). There are, however, different definitions of galactic conformity that can be lead to different quantitative results. In this paper, we focus on the quenched fraction of central galaxies, the same as the conformity definition used by Hearin, Watson \& van den Bosch (2015). Kauffmann et al. (2013) measure conformity by measuring median star formation rates around samples of large isolated galaxies, where the isolated galaxies are divided into many bins based on their specific SFR). Kauffmann et al. (2013) find a strong suppression of sSFR of galaxies around the least star-forming isolated galaxies. The stellar mass range at which Kauffmann et al.
(2013) find conformity is consistent with the stellar masses at which we find a weak trend of $f_{\mathrm{Q}}$ with $\rho$. It is possible to change the mean sSFR without altering $f_{\mathrm{Q}}$, thus further study is required to see if these observations are compatible. Additionally, a separate effect known as small-scale galactic conformity - the properties of satellite galaxies conforming to that of the central galaxy, first detected by Weinmann et al. (2006) - has been confirmed by other studies (Knobel et al. 2015; Kawinwanichakijet al. 2016; Berti et al. 2016).

A robust theory of galaxy formation must be consistent with all of these results listed above: a formation path that yields clear conformity within a dark matter halo, conformity of star formation rates outside of the halo, but limited to no correlation of the quiescent fraction on large-scale environment. A convincing explanation for all these observations will likely combine the influence of dark matter structure formation with complicated astrophysics and phenomena that is independent of halo formation. This series of papers will probe the limits of the influence of dark matter on presentday galaxy properties, separating - and hopefully simplifying - the problem of galaxy formation into those two regimes.

The authors wish to thank Andrew Hearin for useful discussions, as well as the anonymous referee, whose comments significantly improved the paper. We thank Matthew R. Becker for providing the Chinchilla simulation used in this work. The Chinchilla simulation and related analysis were performed using computational resources at SLAC. We thank the SLAC computational team for their consistent support. JLT acknowledges support from NSF grant AST-121189. ARW was supported by a Moore Prize Fellowship through the Moore Center for Theoretical Cosmology and Physics at Caltech and by a Carnegie Fellowship in Theoretical Astrophysics at Carnegie Observatories. CC acknowledges support from NASA grant NNX15AK14G, NSF grant AST-1313280 and the Packard Foundation.

\section{REFERENCES}

Abazajian K. N. et al., 2009, ApJS, 182, 543

Abbas U., Sheth R. K., 2006, MNRAS, 372, 1749

Baldry I. K., Balogh M. L., Bower R. G., Glazebrook K., Nichol R. C., Bamford S. P., Budavari T., 2006, MNRAS, 373, 469

Bamford S. P. et al., 2009, MNRAS, 393, 1324

Behroozi P. S., Wechsler R. H., Wu H.-Y., Busha M. T., Klypin A. A., Primack J. R., 2013, ApJ, 763, 18

Behroozi P. S., Wechsler R. H., Conroy C., 2013, ApJ, 770, 57

Behroozi P. S., Wechsler R. H., Wu H.-Y., 2013, ApJ, 762, 109

Berti A. M., Coil A. L., Behroozi P. S., Eisenstein D. J., Bray A. D., Cool R. J., Moustakas J., 2016, ApJ, 834, 87

Blanton M. R., Moustakas J., 2009, ARA\&A, 47, 159

Blanton M. R., Roweis S., 2007, AJ, 133, 734

Blanton M. R., Eisenstein D., Hogg D. W., Schlegel D. J., Brinkmann J., 2005, ApJ, 629, 143

Blanton M. R. et al., 2005, AJ, 129, 2562

Brinchmann J., Charlot S., White S. D. M., Tremonti C., Kauffmann G., Heckman T., Brinkmann J., 2004, MNRAS, 351, 1151

Campbell D., van den Bosch F. C., Hearin A., Padmanabhan N., Berlind A., Mo H. J., Tinker J., Yang X., 2015, MNRAS, 452, 444

Chabrier G., 2003, PASP, 115, 763

Conroy C., Wechsler R. H., 2009, ApJ, 696, 620

Conroy C., Wechsler R. H., Kravtsov A. V., 2006, ApJ, 647, 201

Croton D. J., Gao L., White S. D. M., 2007, MNRAS, 374, 1303

Dalal N., White M., Bond J. R., Shirokov A., 2008, ApJ, 687, 12

Davis M., Geller M. J., 1976, ApJ, 208, 13

Dressler A., 1980, ApJ, 236, 351

Gao L., White S. D. M., 2007, MNRAS, 377, L5

Gao L., Springel V., White S. D. M., 2005, MNRAS, 363, L66 
Geha M., Blanton M. R., Yan R., Tinker J. L., 2012, ApJ, 757, 85

Hearin A. P., Watson D. F., 2013, MNRAS, 435, 1313

Hearin A. P., Watson D. F., Becker M. R., Reyes R., Berlind A. A., Zentner A. R., 2014, MNRAS, 444, 729

Hearin A. P., Watson D. F., van den Bosch F. C., 2015, MNRAS, 452, 1958

Hogg D. W. et al., 2004, ApJ, 601, L29

Kauffmann G., White S. D. M., Heckman T. M., Ménard B., Brinchmann J., Charlot S., Tremonti C., Brinkmann J., 2004, MNRAS, 353, 713

Kauffmann G., Li C., Zhang W., Weinmann S., 2013, MNRAS, 430, 1447

Kawinwanichakij L. et al., 2016, ApJ, 817, 9

Knobel C., Lilly S. J., Woo J., Kovač K., 2015, ApJ, 800, 24

Kravtsov A. V., Berlind A. A., Wechsler R. H., Klypin A. A., Gottlöber S., Allgood B., Primack J. R., 2004, ApJ, 609, 35

Lacerna I., Padilla N., Stasyszyn F., 2014, MNRAS, 443, 3107

Leauthaud A. et al., 2012, ApJ, 744, 159

Li Y., Mo H. J., Gao L., 2008, MNRAS, 389, 1419

Maller A. H., Berlind A. A., Blanton M. R., Hogg D. W., 2009, ApJ, 691, 394

Mandelbaum R., Wang W., Zu Y., White S., Henriques B., More S., 2016, MNRAS, 457, 3200

Masters K. L. et al., 2010, MNRAS, 404, 792

Moster B. P., Somerville R. S., Maulbetsch C., van den Bosch F. C., Macciò A. V., Naab T., Oser L., 2010, ApJ, 710, 903

Moster B. P., Naab T., White S. D. M., 2013, MNRAS, 428, 3121

Oemler A., Jr, 1974, ApJ, 194, 1

Park C., Choi Y.-Y., Vogeley M. S., Gott J. R. I., Blanton M. R., 2007, ApJ, 658, 898

Reddick R. M., Wechsler R. H., Tinker J. L., Behroozi P. S., 2013, ApJ, 771, 30

Skibba R., Sheth R. K., Connolly A. J., Scranton R., 2006, MNRAS, 369, 68

Springel V., 2005, MNRAS, 364, 1105

Swanson M. E. C., Tegmark M., Blanton M., Zehavi I., 2008, MNRAS, 385, 1635

Swanson M. E. C., Tegmark M., Hamilton A. J. S., Hill J. C., 2008, MNRAS, 387,1391

Tinker J. L., 2016, MNRAS, 467, 3533

Tinker J. L., Weinberg D. H., Zheng Z., Zehavi I., 2005, ApJ, 631, 41

Tinker J. L., Conroy C., Norberg P., Patiri S. G., Weinberg D. H., Warren M. S., 2008, ApJ, 686, 53

Tinker J., Wetzel A., Conroy C., 2011, MNRAS, preprint (arXiv:1107.5046)

Tinker J. L., George M. R., Leauthaud A., Bundy K., Finoguenov A., Massey R., Rhodes J., Wechsler R. H., 2012, ApJ, 755, L5

Tinker J. L., Leauthaud A., Bundy K., George M. R., Behroozi P., Massey R., Rhodes J., Wechsler R. H., 2013, ApJ, 778, 93

Tinker J. L., Leauthaud A., Bundy K., George M. R., Behroozi P., Massey R., Rhodes J., Wechsler R. H., 2013, ApJ, 778, 93

Wang Y., Yang X., Mo H. J., van den Bosch F. C., Weinmann S. M., Chu Y., 2008, ApJ, 687, 919

Wang L., Weinmann S. M., De Lucia G., Yang X., 2013, MNRAS, 433, 515

Wechsler R. H., Bullock J. S., Primack J. R., Kravtsov A. V., Dekel A., 2002, ApJ, 568, 52

Wechsler R. H., Zentner A. R., Bullock J. S., Kravtsov A. V., Allgood B., 2006, ApJ, 652, 71

Weinmann S. M., van den Bosch F. C., Yang X., Mo H. J., 2006, MNRAS, 366,2

Wetzel A. R., Cohn J. D., White M., Holz D. E., Warren M. S., 2007, ApJ, 656,139

Wetzel A. R., Tinker J. L., Conroy C., van den Bosch F. C., 2013, MNRAS, 432, 336

Wetzel A. R., Tinker J. L., Conroy C., Bosch F. C. v. d., 2014, MNRAS, 439, 2687

Yang X., Mo H. J., van den Bosch F. C., Jing Y. P., 2005, MNRAS, 356, 1293

Yang X., Mo H. J., van den Bosch F. C., 2006, ApJ, 638, L55

York D. G. et al., 2000, AJ, 120, 1579

Zehavi I. et al., 2011, ApJ, 736, 59
Zentner A. R., Hearin A. P., van den Bosch F. C., 2014, MNRAS, 443, 3044 Zu Y., Mandelbaum R., 2016, MNRAS, 457, 4360

\section{APPENDIX A: SUPPLEMENTARY HALO AGE DEFINITIONS}

In this Appendix we show results from two more common halo age proxies: the redshift at which half the halo mass forms, $z_{1 / 2}$, and halo concentration, $c_{\mathrm{vir}} \cdot z_{1 / 2}$ is one of the most commonly used halo age definitions in the field, while $c_{\mathrm{vir}}$ is also widely used as a proxy for halo age, given that halo clustering correlates well with $c_{\mathrm{vir}}$ at fixed $M_{\mathrm{h}}$, and that $c_{\mathrm{vir}}$ can be measured for haloes in a single snapshot, without having to create full halo growth histories.

Fig. B1 reprises the assembly bias results show in Fig. 7, only now including the two new halo age proxies listed above. The figure also shows the change in $f_{\mathrm{Q}}^{\text {cen }}$ for the $a_{c}\left(M_{\mathrm{h}}\right)$ age-matching model, for reference. There are slight quantitative differences in the assembly bias induced using these two halo age proxies, but the overall results are in good agreement with the fiducial age definitions used in the main text.

Fig. B2 shows the 'break point' between haloes being classified as 'old' and 'young' for each of our halo age proxies. The use of quotes around these terms is meant to stress that there is possibly no physical significance to these values - they are somewhat arbitrary dividing lines in continuous distributions of halo properties. But it is of interest to document the values required to match the observed values of $f_{\mathrm{Q}}^{\text {cen }}$ as a function of $M_{*}$. The top panel shows the break point when using fractional growth as our age proxy; i.e. haloes of $M_{\mathrm{h}} \sim 10^{11.4} h^{-1} \mathrm{M}_{\odot}$ (which house galaxies of $M_{*} \sim 10^{9.5} \mathrm{M}_{\odot} h^{-2}$ ), contain quenched galaxies if their fractional growth rate is less than 17 per cent, when measured from $z=0.8 \rightarrow 0$. The middle panel shows the results using our halo formation epoch estimates: $a_{c}\left(M_{\mathrm{h}}\right)$, $a_{c}\left(M_{\text {peak }}\right)$ and $z_{1 / 2}$. We note that the formation epoch for haloes using $z_{1 / 2}$ is much smaller than when using $a_{c}$, but the amplitude and nature of the assembly bias is nearly the same. In the bottom panel, we show the values of $c_{\text {vir }}$ that delineate old from young haloes.

We note that the values shown for the $a_{c}$ model in Fig. B2 are significantly smaller than those in the published version of Hearin \& Watson (2013, their fig. 2). An updated version of their fig. 2, which will be submitted as an erratum, are in good agreement with our results (A. Hearin, private communication). We also note that the absolute values of the break points do not alter the rank ordering of the haloes and the results of their paper are unchanged.

\section{APPENDIX B: TESTING SCATTER IN HALO MASSES}

Campbell et al. (2015) demonstrated that the group finder may yield biased values of $f_{\mathrm{Q}}^{\text {cen }}$ where binned in $M_{\mathrm{h}}$ rather than $M_{*}$. This is mainly due to intrinsic scatter in $M_{*}$ at fixed $M_{\mathrm{h}}$. The group finder assigns halo mass to groups based on the rank-order of their total group stellar mass, thus the halo masses will be biased to some degree. Binning a quantity by halo mass, rather than galaxy mass, exacerbates this bias because scatter in halo mass at fixed galaxy mass can be significantly larger than the inverse. This is why we only compare to $f_{\mathrm{Q}}^{\text {cen }}$ when binned in stellar mass.

Fig. B3 demonstrates that our theoretical models are robust to this bias. The blue curves show our fiducial result, using the true halo masses from the simulation. The red curves show a result using halo masses that are biased in the same manner as might arise from the group finder. First, true halo masses are assigned stellar masses 


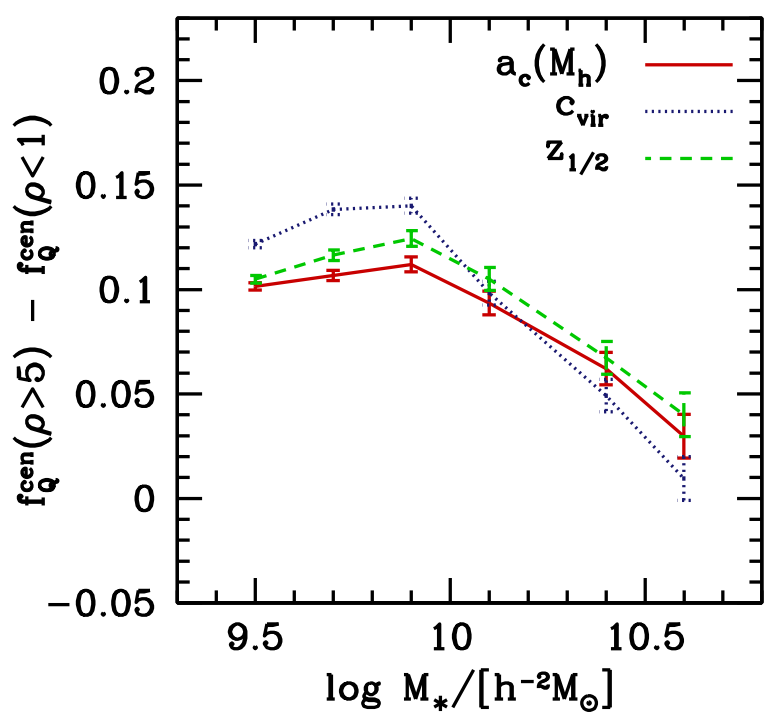

Figure B1. Same as Fig. 7, but now showing the results for halo agematching models in which haloes are rank ordered by $z_{1 / 2}$ and $c_{\mathrm{vir}}$. For comparison, the model that uses $a_{c}\left(M_{\mathrm{h}}\right)$ is also shown.

using the mean relation from Fig. 3. Then 0.2 dex of scatter is added to these $M_{*}$ values. The new, scattered values of $M_{*}$ are re-mapped on to halo mass using the original relationship between halo mass and stellar mass. These are then biased halo masses. The haloes are binned based upon their mass (in this case, the new biased masses), and the age-matching procedure is repeated to obtain a quenched fraction. Although the distribution of true halo masses in this new prediction does not match the distribution of true halo masses from the fiducial model, the impact on $f_{\mathrm{O}}^{\text {cen }}$ is negligible. This is because the mapping of halo age on to $\mathrm{D}_{n} 4000$ is done after the haloes are binned, and because the relationship between halo growth (or halo age) and $M_{\mathrm{h}}$ varies only slowly with halo mass.

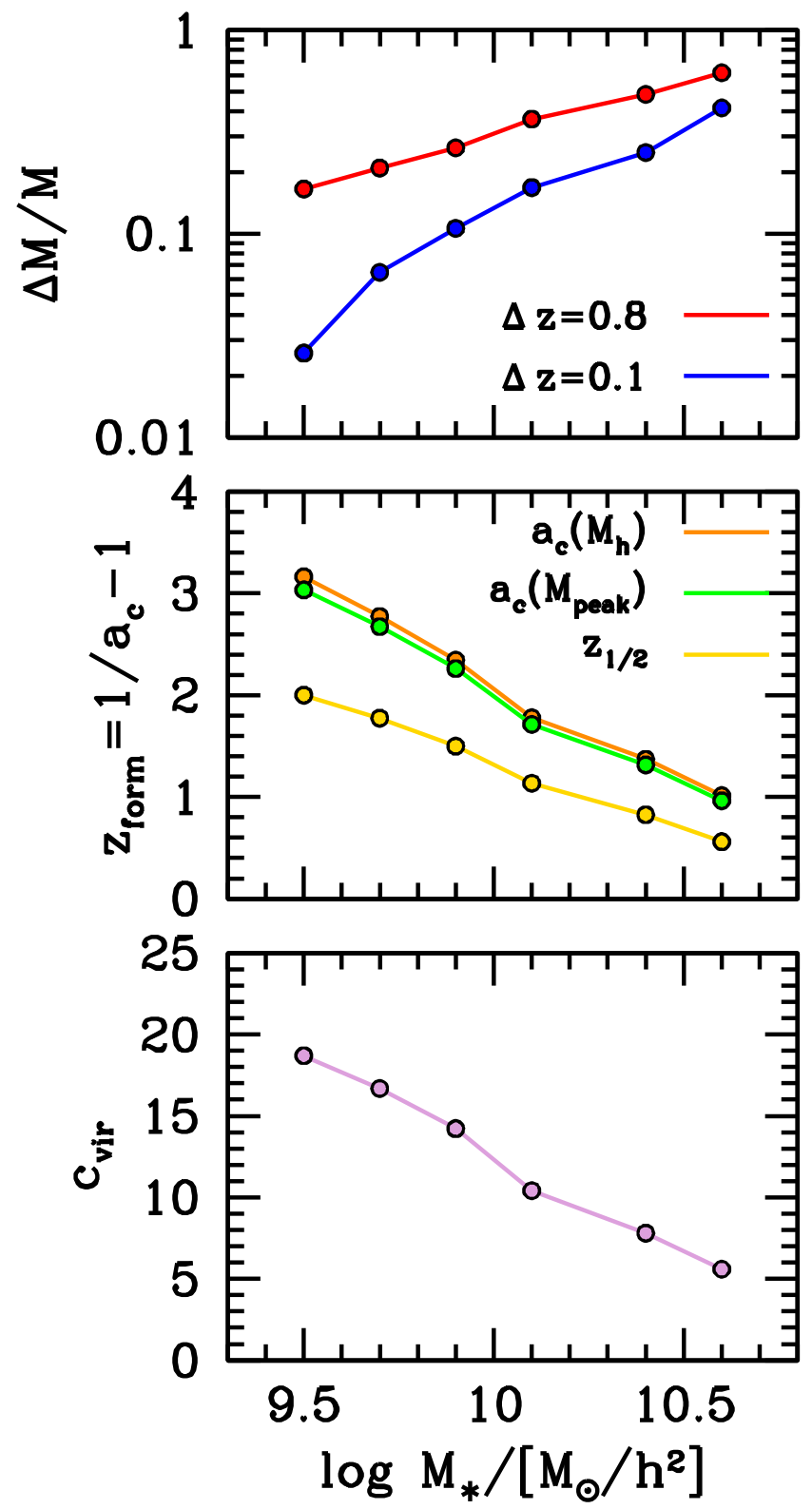

Figure B2. Each panel shows the break point between haloes categorized as 'old' and 'young' for each halo age proxy. For each galaxy stellar mass, the old fraction is set to match the observed quenched fraction for central galaxies. Top panel: break point dividing old and young haloes as determined by their fractional growth. Results are shown for redshift baselines $\Delta z=0.8$ and $\Delta z=0.1$. The trend of rising growth rate with $\log M_{*}$ is due in small part to changing growth rates with halo mass, but mostly due to the rising $f_{\mathrm{Q}}^{\text {cen }}$ with $\log M_{*}$. Middle panel: break point using three different definitions of formation epoch as described in the text. Bottom panel: break point between old and young haloes using $c_{\mathrm{vir}}$ as the age proxy. 


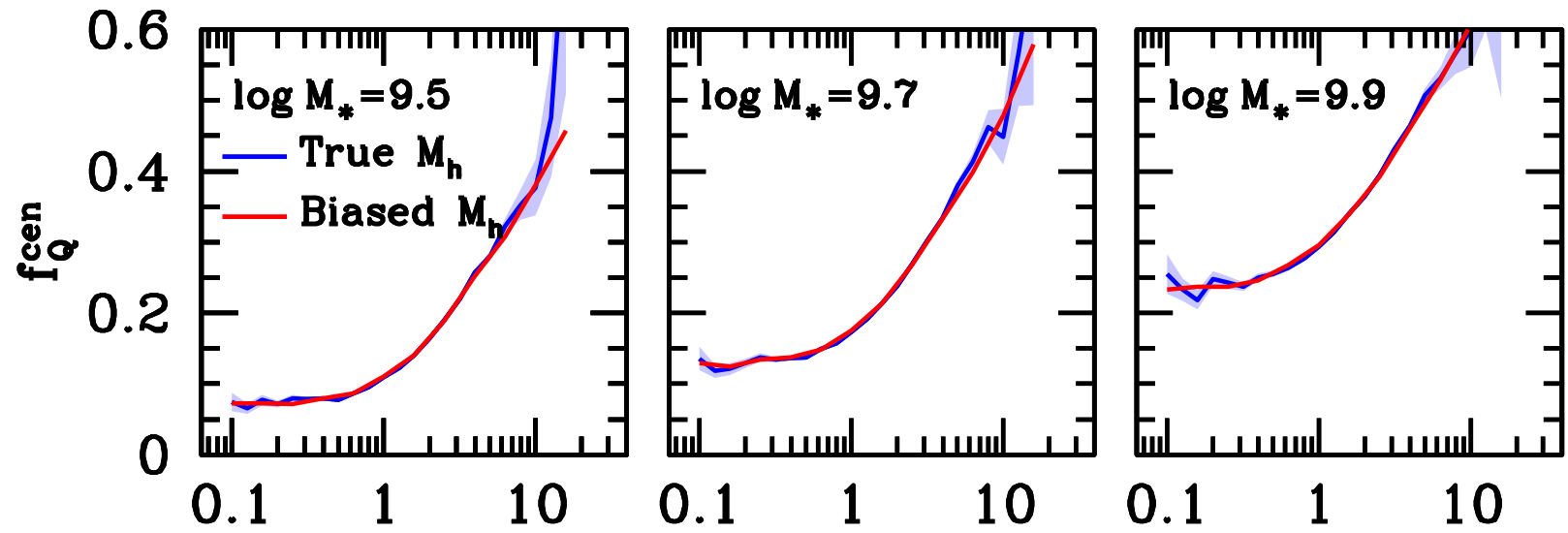

Figure B3. Theoretical predictions for the quenched fraction of halo from the age matching model. The blue curves with shaded errors are from our fiducial model, which uses the true halo mass from the simulation. The red curve uses a biased halo mass. The halo mass is biased in the same way that the group finder can bias $M_{\mathrm{h}}$. But, since the haloes are rank ordered in growth rate after the bias is imparted, there is negligible change in their predicted $f_{\mathrm{Q}}^{\text {cen }}$. This is due to the fact that our age proxy - in this example we use $\Delta z=0.8$ halo growth - changes slowly with halo mass. See the text for further details.

This paper has been typeset from a $\mathrm{TE}_{\mathrm{E}} \mathrm{L} \mathrm{L} \mathrm{T} \mathrm{E} X$ file prepared by the author. 